Teaching Case Report

\section{Recurrent meningitis in a child due to an occult}

\section{spinal lesion}

The case: A 1-year-old girl presented to hospital with fever, lethargy and vomiting. She had had an episode of Escherichia coli meningitis at 2 months of age, which had been treated with antibiotics. No further investigation was conducted at that time. The remainder of her medical history was unremarkable.

On examination, the child was febrile, lethargic and cried with neck flexion. She had a port-wine stain over her lumbar spine at the midline (Fig. 1). Lumbar puncture revealed a leukocyte count of $3340 \times 10^{6} / \mathrm{L}$ (normal value $<5$ $\left.\times 10^{6} / \mathrm{L}\right)$ with $78 \%$ polymorphs, an erythrocyte count of $110 \times 10^{6} / \mathrm{L}$, a glucose level of $1.9 \mathrm{mmol} / \mathrm{L}$ (the serum glucose level was $13.3 \mathrm{mmol} / \mathrm{L}$ ) and a protein level of $0.74 \mathrm{~g} / \mathrm{L}$ (normal $0.15^{-0.40} \mathrm{~g} / \mathrm{L}$ ). Results of blood and urine cultures were negative. Her cerebrospinal fluid tested positive for $E$. coli. In addition,

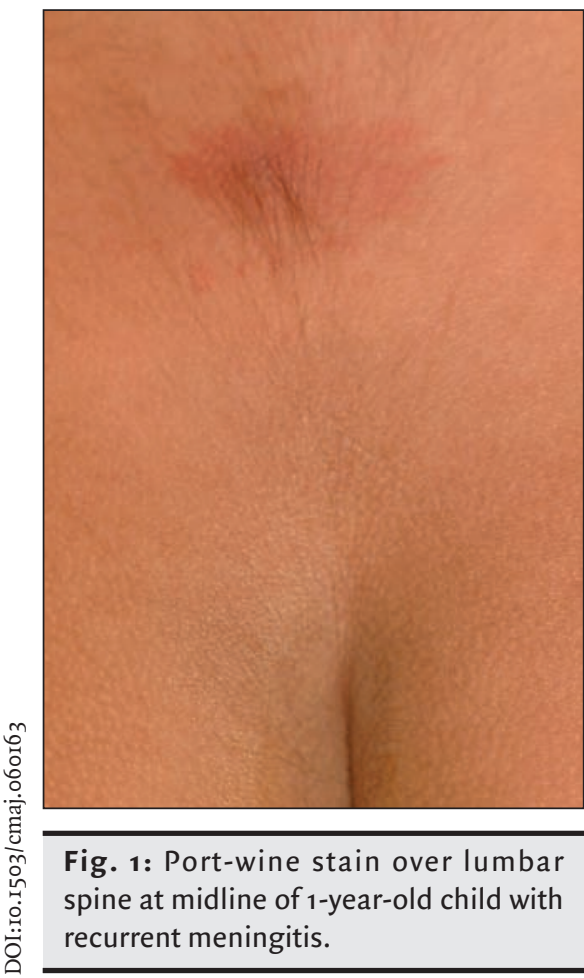

growth of Peptoniphilus asaccharolyticus was noted.

There was no history of recurrent infection or head trauma. Results of tests for HIV infection and immunodeficiency were negative. A spinal MRI scan revealed 3 lesions in the lumbar region compatible with epidermoid or dermoid cysts. A sinus tract extending from the skin into the third extradural lesion was also noted (Fig. 2). On more careful clinical assessment, with rostral and caudal traction of the skin, tethering of the skin was seen supporting the presence of a sinus tract, and an overlying pustule was evident in the centre of the port-wine stain (Fig. 3).

In consultation with infectious disease specialists, a 6-week course of meropenem, amikacin and vancomycin was administered. The child then underwent neurosurgical excision of the sinus tract and resection of the cystic

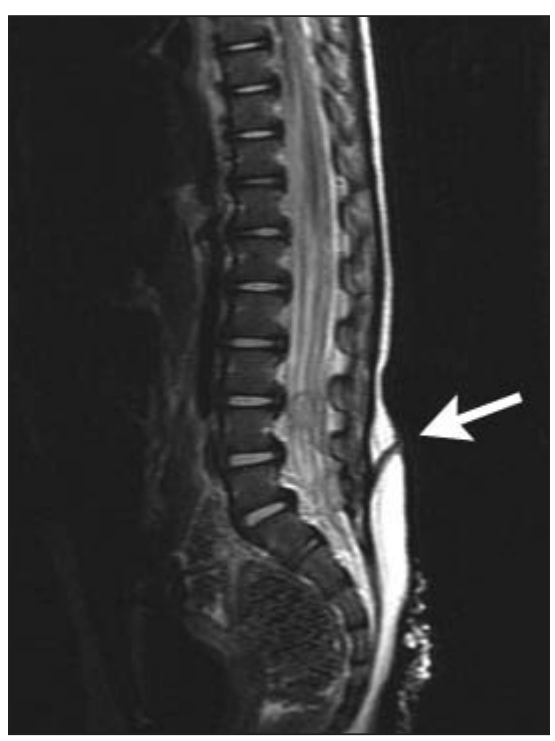

Fig. 2: Spinal MRI scan, showing 3 lesions in lumbar region compatible with epidermoid or dermoid cysts, and sinus tract extending from skin into third extradural lesion. lesions. Pathology revealed the presence of hair elements, which confirmed the diagnosis of dermoid cysts.

Recurrent meningitis can lead to significant morbidity and mortality. Careful history taking, physical examination and investigations are required to rule out causes of recurrent bacterial meningitis (Box I). Examination of a newborn's back is important to check for signs that suggest an underlying spinal defect (Box 2). Possible spinal defects include dermal sinus tracts, intraspinal cysts, intraspinal lipomas or other tumours, and tethered spinal cord. These abnormalities may lead to progressive weakness or sensory loss in the lower extremities, gait abnormalities, bowel and bladder dysfunction, and foot deformities.

More than $90 \%$ of patients with an occult spinal abnormality have a cutaneous marker over the lower spine. However, if only I cutaneous marker is present, spinal defects are found in less than $\mathrm{r} \%$ of patients. The presence of 2 or more cutaneous markers increases the likelihood of an occult spinal abnormality. ${ }^{1}$ A coccygeal pit (Fig. 4) is not associated with any intradural pathology and should be differentiated from a sacral dimple. Coccygeal pits are small, less than $5 \mathrm{~mm}$ in diameter, are located in the midline within 2.5

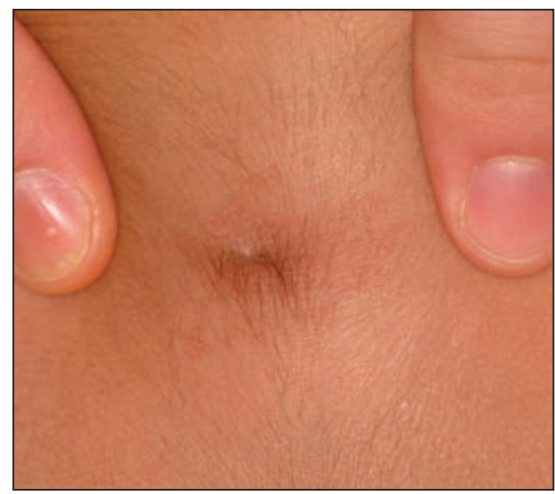

Fig. 3: Rostral and caudal traction of skin revealed presence of sinus tract and overlying pustule in centre of portwine stain. 
Box 1: Causes of recurrent bacterial meningitis

Immunodeficiency

Congenital

- B-cell or combined B- and T-cell defect, complement deficiency, asplenia

Acquired

- Splenectomy, HIV infection

Central nervous system abnormality

Congenital

- Intracranial or intraspinal defects with or without a dermal connection, skull base defects, inner ear defects, neuroenteric fistula

Acquired

- Skull fracture, shunt or instrumentation, chronic contiguous infection

Box 2: Markers that may suggest a spinal defect

- Tuft of hair

- Sacral dimple

- Vascular marking (e.g., port-wine stain)

- Sinus ostium*

- Skin tag over spine

- Subcutaneous lipoma over spine

- Local erythema or induration suggesting infection

*Small opening that leads to a deep sinus tract. $\mathrm{cm}$ of the anus and may be present in up to $4 \%$ of the normal population. ${ }^{1,3}$ Sacral dimples, on the other hand, are usually larger than $5 \mathrm{~mm}$ in diameter, are located above the intergluteal cleft and are often found with other cutaneous markers. Current recommendations state that spinal ultrasounds should be performed in infants less than 4-6 months of age with any cutaneous marker over the spine. ${ }^{4}$

All newborns should have a careful physical examination, which includes

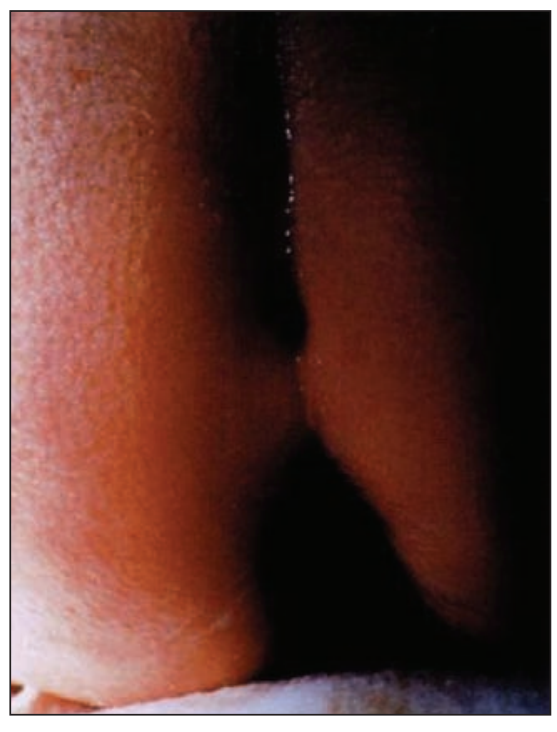

Fig. 4: Simple midline dimple (coccygeal pit) in newborn. Coccygeal pits are not associated with any intradural pathology. Reproduced, with permission, from Kriss et al. their back. If a cutaneous abnormality is seen, rostral and caudal traction of the skin may identify the presence of a sinus tract. The ease and availability of spinal ultrasound makes it a valuable screening tool. If an abnormality is found or the test result is unclear, then an MRI scan is recommended. Nonurgent referral to a neurosurgeon can be made if the results of neurologic examination are normal. An urgent referral must be made if an infection is present or neurologic deficits are detected.

\section{Hosanna Au}

Department of Paediatrics

Faculty of Medicine

University of Toronto

Hospital for Sick Children

Toronto, Ont.

Competing interests: None declared.

Acknowledgements: I thank Dr. Jeremy Friedman for his thoughtful review of the initial draft of the manuscript and Dr. Peter Dirks for his teaching and the photographs.

\section{REFERENCES}

I. Guggisberg D, Hadj-Rabia S, Viney C, et al. Skin markers of occult spinal dysraphism. Arch Dermatol 2004;I40:IIo9-I5.

2. Kriss VM, Desai NS. Occult spinal dysraphism in neonates: assessment of high-risk cutaneous stigmata on sonography. Am J Roentgenol 1998;171: I687-92.

3. Ackerman LL, Menezes AH. Spinal congenital dermal sinuses: a 30-year experience. Pediatrics 2003;II2:64I-7.

4. Hughes JA, De Bruyn R, Patel K, et al. Evaluation of spinal ultrasound in spinal dysraphism. Clin Radiol 2003;58:227-33. 